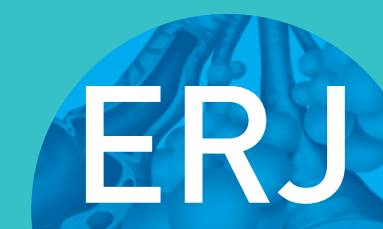

open research

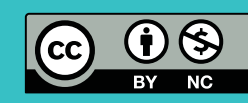

\section{Patterns, predictors and outcomes of asthma control and exacerbations during pregnancy: a prospective cohort study}

\author{
Luke E. Grzeskowiak ${ }^{1}$, Brian Smith ${ }^{2}$, Anil Roy ${ }^{2}$, Gustaaf A. Dekker ${ }^{1}$ and \\ Vicki L. Clifton ${ }^{1,3}$
}

Affiliations: ${ }^{1}$ Robinson Research Institute, School of Paediatrics and Reproductive Health, University of Adelaide, Adelaide, Australia. ${ }^{2}$ Respiratory Medicine Unit, The Queen Elizabeth Hospital, Adelaide, Australia. ${ }^{3}$ Mater Medical Research Institute, University of Queensland, Brisbane, Australia.

Correspondence: Vicki L. Clifton, Mater Medical Research Institute, Level 4, Translational Research Institute, 37 Kent St, Woolloongabba, Qld. 4101, Brisbane, Australia. E-mail: Vicki.cliftonamater.uq.edu.au

ABSTRACT There exists a paucity of data for socially disadvantaged populations describing patterns and predictors of asthma control status and exacerbations during pregnancy, and their relationship to adverse perinatal outcomes.

Asthmatic women $(\mathrm{n}=189)$ were followed prospectively during pregnancy, with visits at 12, 20, 28 and 36 weeks gestation. Data on loss of control, recurrent uncontrolled asthma and moderate/severe exacerbations were collected at each visit and their relationship to perinatal outcomes examined following stratification for fetal sex.

$50 \%$ of asthmatic women experienced a loss of control or moderate/severe exacerbation during pregnancy, with $22 \%$ of women experiencing a moderate/severe exacerbation. Factors associated with an increased risk of women experiencing recurrent uncontrolled asthma during pregnancy included smoking (relative risk 2.92, 95\% CI 1.53-5.58), inhaled corticosteroid use at the beginning of pregnancy (relative risk 2.40, 95\% CI 1.25-4.60) and increasing maternal age (relative risk 1.06, 95\% CI 1.01-1.11). No factors were associated with moderate/severe exacerbations. Asthma control rather than exacerbations during pregnancy appeared to be most strongly correlated with perinatal outcomes. Following stratification by fetal sex, the presence of recurrent uncontrolled asthma was associated with an increased risk of being small for gestational age in women pregnant with females ( $33.3 \%$ versus $9.5 \% ; \mathrm{p}=0.018)$. In contrast, there was a nonsignificant increased risk of preterm birth in women with recurrent uncontrolled asthma that were pregnant with males $(25.0 \%$ versus $11.8 \%$; $=0.201)$

These results suggest that the key to improving perinatal outcomes lies in improving asthma control as early as possible in pregnancy and monitoring throughout pregnancy, rather than focusing on preventing exacerbations alone.

@ERSpublications

Recurrent uncontrolled asthma during gestation is a greater contributor to poor perinatal outcomes than exacerbations http://ow.ly/X0OSa

Received: Aug 102015 | Accepted after revision: Dec 152015

Support statement: The project was funded by the University of Adelaide. Salary support was provided through a National Health and Medical Research Council Senior Research Fellowship (APP1041918 to V.L. Clifton) and National Health and Medical Research Council Australian Public Health Fellowship (APP1070421 to L.E. Grzeskowiak). Funding information for this article has been deposited with FundRef.

Conflict of interest: None declared.

Copyright $\odot$ ERS 2016. This article is open access and distributed under the terms of the Creative Commons Attribution Non-Commercial Licence 4.0. 


\section{Introduction}

The adverse effects of asthma in pregnancy are related to asthma severity and the intensity of treatment [1-3]. Acute asthma exacerbations have been identified as the most significant event to affect fetal morbidity and mortality in pregnancies complicated by asthma $[4,5]$. A number of studies have examined the effects of maternal asthma during pregnancy, in the presence and absence of inhaled corticosteroid (ICS) treatment, on placental function and fetal development [6]. These studies have demonstrated that use of ICS for the treatment of asthma did not affect fetal growth, and that maternal asthma without treatment had a greater impact on the fetus and placenta [7]. Overall, the evidence clearly demonstrates no increased risk of adverse maternal or fetal complications in situations where asthma is well controlled throughout pregnancy $[8,9]$. In contrast, poor asthma control, as indicated by the need for more intensive treatment, is a greater risk factor for an adverse outcome during pregnancy than ICS use [5]. Further, regular use of oral corticosteroids (OCS), used in the management of acute asthma exacerbations and poorly controlled asthma during pregnancy, has been associated with an increased incidence of low birth weight babies and preterm birth [10]. These findings highlight the importance of optimising asthma control and reducing asthma exacerbations.

Previous studies have defined asthma exacerbations as any asthma-related event that involved one or more of the following: a hospital admission, an unscheduled visit to a doctor, a course of oral steroids, an increase in medication use or decreased peak expiratory flow rate. Among those with mild, moderate and severe asthma the exacerbation rates have been reported as $13 \%, 26 \%$ and $52 \%$, respectively [11]. The corresponding rates of exacerbations leading to hospitalisation were $2 \%, 7 \%$ and $27 \%$ [11]. Relying solely on asthma severity, however, can be misleading as asthma can worsen during pregnancy regardless of maternal pre-pregnancy severity $[4,5]$. Exacerbations can occur at any time during gestation, but are most likely in the second and third trimesters between weeks 17 and 34, with peak incidence at around 25 weeks gestation. Many interventions for pregnant asthmatic women are focused on reducing asthma exacerbations as it is likely to have the greatest impact on improving perinatal outcomes [12].

A major comorbidity of pregnant women with asthma is cigarette use [13, 14]. We previously found that $25 \%$ of asthmatic women smoked during pregnancy relative to $17 \%$ of non-asthmatic women [14]. Asthmatic women who were current smokers during pregnancy had more severe asthma exacerbations during pregnancy relative to women who were nonsmokers [15]. The combination of asthma and cigarette use also significantly increased the incidence of low birth weight neonates and preterm deliveries [14].

It is recognised that social disadvantage encompasses a range of contributing factors including health literacy, income, occupation, housing, health behaviours (i.e. smoking and poor diet) and comorbidities (i.e. mental health) as well as race and/or ethnicity. The prevalence of asthma is greatest among those living in lower socioeconomic status areas, with low socioeconomic status also associated with almost double the rate of smoking, increased rates of hospitalisations for asthma and an increased risk of asthma-related mortality [16]. Children from low socioeconomic status communities are more likely to have poorly controlled asthma relative to children of high socioeconomic status [17] and it has been proposed that socioeconomic status index is a strong predictor of poor asthma outcomes. The BIOAIR study demonstrated that low socioeconomic status is a risk factor for exacerbations [18]. However, poor asthma control and exacerbations in low socioeconomic status populations may be due to inadequacies in health literacy [19]. APTER et al. [20] reported that reduced literacy and numeracy skills in association with ethnicity, age, income and educational attainment were all associated with level of asthma control, with those individuals with reduced literacy being more likely to have uncontrolled asthma.

In the current study, we have examined asthma control and exacerbations in a group of pregnant women who live in a socially disadvantaged community in South Australia. The level of social deprivation in this pregnant population is significant. For example, $36 \%$ of pregnant women from this population have reported they were abused as children, 35\% have suffered major life stressors and $30 \%$ of these pregnant women were diagnosed with depression during antenatal care [21]. Population-based data report that $40 \%$ of the inhabitants in this community do not finish year 10 of high school, $>22 \%$ of the population are unemployed, $27 \%$ are housed by the government and $22 \%$ of families have a sole female parent [22]. Our objective was to examine patterns and predictors of asthma control and exacerbations during pregnancy in a population of women from a socially disadvantaged community, and to determine the association of this data with perinatal outcomes.

\section{Methods}

Approval for the study was provided by The Queen Elizabeth Hospital, Lyell McEwin Hospital and Modbury Hospital Human Research Ethics Committee and The University of Adelaide Human Research Ethics Committee. All women gave written informed consent. 
Prospective asthma study

Pregnant women with a doctor diagnosis of asthma $(n=189)$ were recruited through the Lyell McEwin Hospital antenatal clinics (South Australia, Australia) between May 2009 and May 2012 to take part in a non-interventional prospective cohort study evaluating the impact of maternal asthma on perinatal outcomes. Women were assessed by a midwife, with additional respiratory training, at $12,20,28$, and 36 weeks gestation. The midwife utilised a standardised data collection tool to collect demographic data including maternal weight, height, previous obstetric history and medical history. Maternal smoking was assessed by maternal self-report, with women classified according to smoking status during the first antenatal visit. Socioeconomic status for each woman was determined using her residential postcode at the time of delivery. Women were then ranked according to their level of advantage, or relative disadvantage, based on data from the Socio-Economic Indexes for Areas, calculated from the Australian Bureau of Statistics' 5-yearly Census of Population and Housing. These indexes are widely used measures of relative socioeconomic status [2]. In addition to demographic variables, data were collected to determine current asthma therapy and control, current asthma triggers, comorbidities and past history including frequency of OCS use and hospital admissions for asthma. Asthma control was assessed using the Asthma Control Questionnaire (ACQ). The ACQ is a seven-item validated questionnaire which covers asthma symptoms and lung function, with scores $>1.5$ indicative of uncontrolled asthma [23]. Forced expiratory volume in $1 \mathrm{~s}\left(\mathrm{FEV}_{1}\right)$ and forced vital capacity (FVC) were measured using an Easy One spirometer (ndd Medical Technologies, Inc., Andover, MA, USA) before and after administration of salbutamol. FEV1 \% predicted was calculated based on the patient's age, ethnicity and height using the equations of Gore et al. [24]. Data were prospectively collected on asthma medications (dose, frequency and duration across trimesters). The same midwife saw women for each of their respective visits. Patients assessed as unstable and requiring medical review were referred to their primary care physician or to a respiratory physician.

\section{Maternal asthma and perinatal outcomes}

Details of the exacerbations were obtained from the study visit notes and confirmed by consulting the medical record if necessary. An exacerbation event was defined as moderate/severe if it required a hospital admission, emergency department presentation, an unscheduled visit to a doctor or a course of oral steroids due to asthma. A loss of control was defined as an increase in asthma symptoms or an increase in medication use, which was managed by the subject and did not require medical intervention, or an ACQ score of $>1.5$ during an asthma study visit. Most women were identified as having a loss of control using the ACQ score at a study visit. An additional asthma outcome included recurrent uncontrolled asthma, defined as an ACQ score of $>1.5$ during two or more study visits. Data were prospectively collected at each study visit. Data on perinatal outcomes were collected at delivery from the medical records, including infant sex, gestational age, birthweight, birth length, head circumference, method of delivery and Apgar scores. Gestational age was determined by the date of the last menstrual period and confirmed by ultrasound at 18 weeks. Preterm birth was defined as $<37$ completed weeks gestation, while small for gestational age (SGA) was defined according to birthweight ( $<10$ th percentile for gestational age).

\section{Statistical analysis}

The impact of various maternal characteristics on asthma exacerbations and recurrent uncontrolled asthma during pregnancy were examined using a generalised linear model, generating relative risks and 95\% confidence intervals. The impact of asthma exacerbations and recurrent uncontrolled asthma on perinatal outcomes were examined using unpaired t-tests for parametric continuous variables, and Fisher's exact test for categorical variables, following stratification for infant sex. All reported p-values were two-tailed, and a p-value of $<0.05$ was considered to be statistically significant. All statistical procedures were carried out using SPSS version 19 (SPSS Inc., Chicago, IL, USA).

\section{Results}

Pregnant women with asthma $(n=189)$ were more likely to be overweight $(24 \%)$ or obese (35\%) than of normal weight (40\%). They were predominantly Caucasian and in the lowest two quintiles for socioeconomic status (85\%). One third of women were treated with an ICS during pregnancy and $78 \%$ of those women were using a combination drug of an ICS with a long-acting $\beta_{2}$-agonist. $54 \%$ of asthmatic women did not use an ICS during pregnancy and 13.8\% of women were prescribed an ICS during pregnancy. $20 \%$ of asthmatics were current smokers. Overall, $22 \%$ of women experienced a moderate/severe exacerbation during pregnancy, $50 \%$ of women had a loss of control during gestation and $16 \%$ had recurrent uncontrolled asthma as indicated by more than two episodes where the ACQ score was $>1.5$ (table 1).

The percentage of women who experienced an exacerbation or loss of control (figure 1a) increased linearly as gestation progressed. Similarly, the percentage of exacerbations and loss of control also increased as gestation progressed (figure $1 \mathrm{~b}$ ), which was comparable to the number of exacerbations or loss of control 


\begin{tabular}{|c|c|}
\hline Subjects $\mathrm{n}$ & 189 \\
\hline Maternal age years & $26.1 \pm 5.5$ \\
\hline $\mathrm{BMI} \mathrm{kg} \cdot \mathrm{m}^{-2}$ & $28.4 \pm 7.2$ \\
\hline \multicolumn{2}{|l|}{ BMI category } \\
\hline Normal weight & 76 (40.2) \\
\hline Overweight & $46(24.3)$ \\
\hline Obese & $67(35.4)$ \\
\hline Weight gain during pregnancy kg & $10.7 \pm 6.5$ \\
\hline \multicolumn{2}{|l|}{ Socioeconomic status } \\
\hline 1 (Lowest) & 100 (52.9) \\
\hline 2 & 61 (32.3) \\
\hline 3 & 6 (3.2) \\
\hline 4 & $12(6.3)$ \\
\hline 5 (Highest) & $10(5.3)$ \\
\hline \multicolumn{2}{|l|}{ Ethnicity } \\
\hline Caucasian & 176 (93.1) \\
\hline Other & $13(6.9)$ \\
\hline \multicolumn{2}{|l|}{ Parity } \\
\hline$\geqslant 1$ & $111(58.7)$ \\
\hline \multicolumn{2}{|l|}{ Smoking status } \\
\hline Never/former smoker & 128 (67.7) \\
\hline Quit smoking during pregnancy & $23(12.2)$ \\
\hline Current smoker & $38(20.1)$ \\
\hline \multicolumn{2}{|l|}{ Baseline ICS use } \\
\hline Yes & 61 (32.3) \\
\hline No & $128(67.7)$ \\
\hline \multicolumn{2}{|l|}{ Baseline ICS type } \\
\hline ICS & 13 (21.3) \\
\hline $\mathrm{ICS}+\mathrm{LABA}$ & 48 (78.7) \\
\hline \multicolumn{2}{|l|}{ Spirometry } \\
\hline FEV 1 L & $3.02 \pm 0.43$ \\
\hline $\mathrm{FEV}_{1} \%$ pred & $92 \pm 11$ \\
\hline FVC L & $3.70 \pm 0.56$ \\
\hline $\mathrm{FEV}_{1} / \mathrm{FVC}$ & $0.82 \pm 0.06$ \\
\hline \multicolumn{2}{|l|}{ New ICS in pregnancy } \\
\hline No & $102(54)$ \\
\hline Yes & $26(13.8)$ \\
\hline Using ICS pre-pregnancy & $61(32.3)$ \\
\hline Any severe exacerbation & $41(21.7)$ \\
\hline OCS use & $14(7.4)$ \\
\hline General practitioner visit & $31(16.4)$ \\
\hline Emergency department visit & $10(5.3)$ \\
\hline Admitted to hospital & $2(1.1)$ \\
\hline Persistent uncontrolled asthma & $30(15.9)$ \\
\hline
\end{tabular}

Data are presented as mean \pm SD or $\mathrm{n}(\%)$, unless otherwise stated. BMI: body mass index; ICS: inhaled corticosteroid; LABA: long-acting $\beta_{2}$-agonist; FEV1: forced expiratory volume in $1 \mathrm{~s}$; FVC: forced vital capacity; OCS: oral corticosteroid.

throughout gestation for the entire population (figure 1c). Overall there were more asthmatic women identified with a loss of asthma control (50\%) as gestation progressed than with an exacerbation (22\%).

When examining the relative risk of a severe exacerbation there were no significant maternal characteristics that contributed to an exacerbation during pregnancy (table 2). However, maternal age, normal body mass index (BMI) and quitting smoking appeared protective against an exacerbation during pregnancy. Baseline ICS was a nonsignificant predictor of exacerbation risk. The presence of an asthma exacerbation during pregnancy was not associated with any statistically significant increase in adverse perinatal outcomes (table 3).

Interestingly, there were significant associations between maternal characteristics and the presence of recurrent uncontrolled asthma in this cohort (table 4). Women with uncontrolled asthma were older (relative risk 1.06, 95\% CI 1.01-1.11), overweight (relative risk 1.89, 95\% CI 0.89-4.04), current smokers (relative risk 2.92, 95\% CI 1.53-5.58) and prescribed an ICS (possibly a reflection of asthma severity) (relative risk 2.40, 95\% CI 1.25-4.60) (table 4). Following stratification by fetal sex, the presence of recurrent uncontrolled 

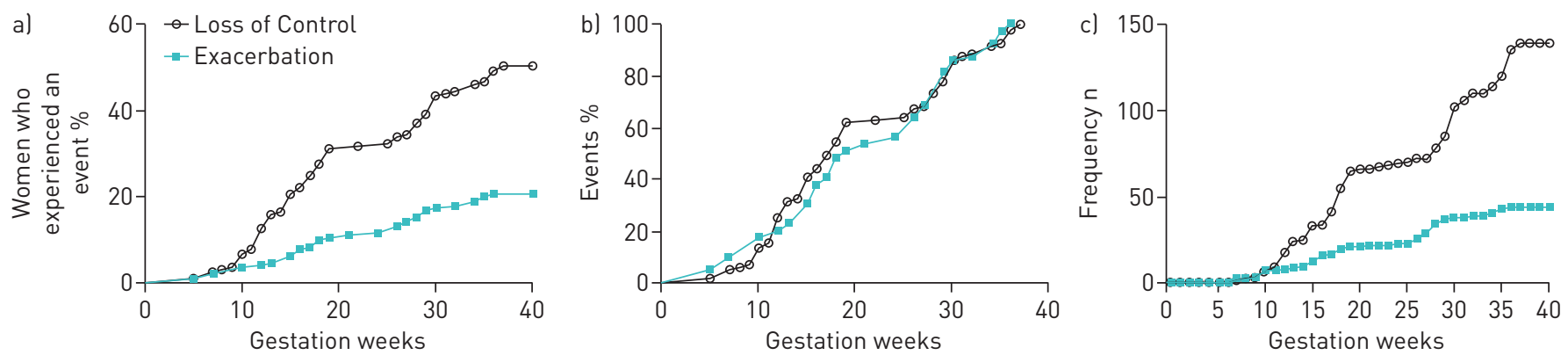

FIGURE 1 Examination of the prevalence of asthma exacerbations and loss of asthma control throughout pregnancy in women with asthma. A loss of control was defined as an increase in asthma symptoms or an increase in medication use, which was managed by the subject and did not require medical intervention, or an Asthma Control Questionnaire score of >1.5 during an asthma study visit. An exacerbation event was defined as moderate/severe if it required a hospital admission, emergency department presentation, an unscheduled visit to a doctor or a course of oral steroids due to asthma. a) The percentage of women who experienced an exacerbation or loss of control as gestation progressed. b) The percentage of all asthma exacerbations or losses of control according to the number of weeks of gestation. c) The cumulative number of exacerbations or losses of control as gestation progressed within the entire population.

asthma at two or more study visits was associated with an increased risk of SGA (33.3\% versus 9.5\%; $\mathrm{p}=0.018)$, but not preterm birth $(11.1 \%$ versus $9.5 \% ; \mathrm{p}=1.000)$ among women pregnant with females (table 5). By contrast, a nonstatistically significant increased risk of preterm birth was observed for males ( $25.0 \%$ versus $11.8 \%$; $\mathrm{p}=0.201$ ), but not SGA ( 8.3 versus $11.8 \% ; \mathrm{p}=1.000$ ) (table 5 ).

\section{Discussion}

This research identified that asthma worsened as gestation progressed in a linear fashion with the greater proportion of women experiencing a loss of control rather than an exacerbation. The women who had recurrent losses of control during pregnancy were significantly more likely to have a poor perinatal outcome than the women who exacerbated during pregnancy, suggesting long periods of uncontrolled asthma may be more harmful than acute exacerbations for the fetus specifically in relation to fetal growth. Interestingly, half of all exacerbations and recurrent losses of control occurred before 20 weeks of gestation, indicating that peri-conceptional interventions and early diagnosis of pregnancy followed by the immediate introduction of asthma education and self-management skills may be essential for preventing the deterioration of asthma with pregnancy.

\begin{tabular}{|c|c|c|c|c|}
\hline & Exacerbation & No exacerbation & p-value & Relative risk $(95 \% \mathrm{CI})$ \\
\hline Subjects $\mathrm{n}$ & 41 & 148 & & \\
\hline Maternal age years & $25.9 \pm 5.8$ & $26.2 \pm 5.6$ & 0.795 & $0.99(0.95-1.04)$ \\
\hline BMI $\mathrm{kg} \cdot \mathrm{m}^{-2}$ & $29.1 \pm 7.5$ & $28.3 \pm 7.2$ & 0.536 & $1.01(0.98-1.05)$ \\
\hline BMI category & & & 0.701 & \\
\hline Normal weight & 17 (41.5) & 59 (39.9) & & Reference \\
\hline Overweight & $8(19.5)$ & $38(25.7)$ & & $0.78(0.37-1.66)$ \\
\hline Obese & $16(39.0)$ & 51 (34.5) & & $1.07(0.59-1.94)$ \\
\hline $\begin{array}{l}\text { Weight gain during pregnancy kg } \\
\text { Parity }\end{array}$ & $9.8 \pm 7.2$ & $11.0 \pm 6.5$ & $\begin{array}{l}0.273 \\
0.699\end{array}$ & $0.98(0.93-1.02)$ \\
\hline 0 & $18(43.9)$ & 60 (40.5) & & $0.90(0.52-1.55)$ \\
\hline$\geqslant 1$ & $23(56.1)$ & 88 (59.5) & & \\
\hline Smoking status & & & 0.557 & \\
\hline Never/former smoker & 29 (70.7) & 99 (66.9) & & Reference \\
\hline Quit smoking during pregnancy & $3(7.3)$ & 20 (13.5) & & $0.58(0.19-1.73)$ \\
\hline Current smoker & $9(22.0)$ & $29(19.6)$ & & $1.05(0.54-2.01)$ \\
\hline Baseline ICS use & & & 0.155 & \\
\hline Yes & 17 (41.5) & $44(29.7)$ & & $1.49(0.87-2.55)$ \\
\hline No & 24 (58.5) & 104 (70.3) & & \\
\hline
\end{tabular}

Data are presented as mean \pm SD or $\mathrm{n}(\%)$, unless otherwise stated. BMI: body mass index; ICS: inhaled corticosteroid. 
TABLE 3 Perinatal characteristics of pregnancies complicated by a moderate or severe exacerbation

\begin{tabular}{|c|c|c|c|c|c|c|}
\hline & \multicolumn{3}{|c|}{ Male } & \multicolumn{3}{|c|}{ Female } \\
\hline Subjects $n$ & 24 & 73 & & 17 & 75 & \\
\hline Gestational age days & $277 \pm 12$ & $270 \pm 20$ & 0.108 & $277 \pm 12$ & $273 \pm 12$ & 0.288 \\
\hline Birthweight $\mathbf{g}$ & $3496 \pm 461$ & $3323 \pm 758$ & 0.294 & $3461 \pm 460$ & $3291 \pm 591$ & 0.271 \\
\hline Birthweight centile & $47.9 \pm 32.4$ & $48.3 \pm 31.2$ & 0.954 & $57.4 \pm 26.2$ & $45.6 \pm 30.5$ & 0.143 \\
\hline Delivery method & & & 0.560 & & & 0.756 \\
\hline Emergency LSCS & $7(29.2)$ & $14(19.2)$ & & $3(17.6)$ & 10 (13.3) & \\
\hline Labour onset & & & 0.772 & & & 1.000 \\
\hline SOL & 14 (58.3) & $37(50.7)$ & & 9 (52.9) & 40 (53.3) & \\
\hline $\mathrm{IOL}$ & $6(25.0)$ & $24(32.9)$ & & 6 (35.3) & $23(30.7)$ & \\
\hline None & $4(16.7)$ & $12(16.4)$ & & $2(11.8)$ & $12(16.0)$ & \\
\hline Birth length $\mathrm{cm}$ & $50.2 \pm 2.1$ & $49.7 \pm 3.9$ & 0.557 & $50.1 \pm 1.9$ & $49.0 \pm 2.2$ & 0.069 \\
\hline SGA & & & 0.726 & & & 1.000 \\
\hline No & 22 (91.7) & 64 (87.7) & & 15 (88.2) & 64 (85.3) & \\
\hline Yes & 2 (8.3) & 9 (12.3) & & $2(11.8)$ & $11(14.7)$ & \\
\hline \multicolumn{7}{|l|}{ Apgar Score } \\
\hline $1 \mathrm{~min}$ & $8 \pm 2$ & $8 \pm 1$ & 0.967 & $8 \pm 1$ & $8 \pm 2$ & 0.191 \\
\hline $5 \mathrm{~min}$ & $9 \pm 1$ & $9 \pm 1$ & 0.449 & $9 \pm 1$ & $9 \pm 1$ & 0.700 \\
\hline
\end{tabular}

We observed that $20 \%$ of women experienced an exacerbation during pregnancy which is in line with the prevalence of exacerbations in mixed socioeconomic status populations of pregnant women [10]. More than $50 \%$ of all exacerbations occurred by 20 weeks of gestation, which is interesting given that most studies of pregnancy, including our previous projects, do not recruit women until later in gestation (>18 weeks gestation) $[7,12]$ and miss an important early time point for intervention and maintaining asthma control. Our previous work in a mixed socioeconomic status population of pregnant asthmatic women observed a decrease in the frequency of exacerbations after 34 weeks gestation [5], but this may be due to study design rather than a real decrease over gestation. The current data suggest that the risk of poor asthma control and exacerbations continues until the end of pregnancy. This may be an effect specific to this particular population or it could be due to a major strength of this particular study in collecting data on asthma outcomes up until delivery.

Loss of control and in particular recurrent loss of control as measured at each visit appears to have a greater impact on perinatal outcome in this population of women. Aside from all the problems associated with social disadvantage, the risks for a loss of control were age, BMI, cigarette use and ICS use, which are similar to previous studies examining risk factors associated with exacerbations [5]. A population-based study of 4434 asthmatic women examined ICS treatment pre-pregnancy and during pregnancy [25]. It was found that $50 \%$ of all women stopped or reduced their ICS intake with pregnancy and $8.2 \%$ exacerbated with cessation, while $20 \%$ of all other women exacerbated. Women using ICS were more likely to exacerbate, which is due to the confounder of asthma severity [25]. Severe asthma increases the requirement for ICS treatment and asthma severity increases the risk of an exacerbation. In our cohort, women with recurrent loss of control are probably similar to findings from previous studies [25] in that ICS use is a reflection of asthma severity, and therefore, they are more susceptible to worsening disease in pregnancy especially if there is a lack of compliance with medication. Previous work has demonstrated that cigarette use in pregnancy is associated with worsening asthma and an increased incidence of exacerbations [15], which may also apply to an increased risk of loss of control as demonstrated in this study. Age being a risk factor is interesting, but may be an interaction with asthma and smoking as the combination of these factors are known to contribute to more airway obstructions with age [26].

There was a high prevalence of overweight and obese women in the current study population, which is in line with state wide prevalence. There is some data to suggest overweight and obesity is associated with 
TABLE 4 Maternal characteristics associated with recurrent uncontrolled asthma

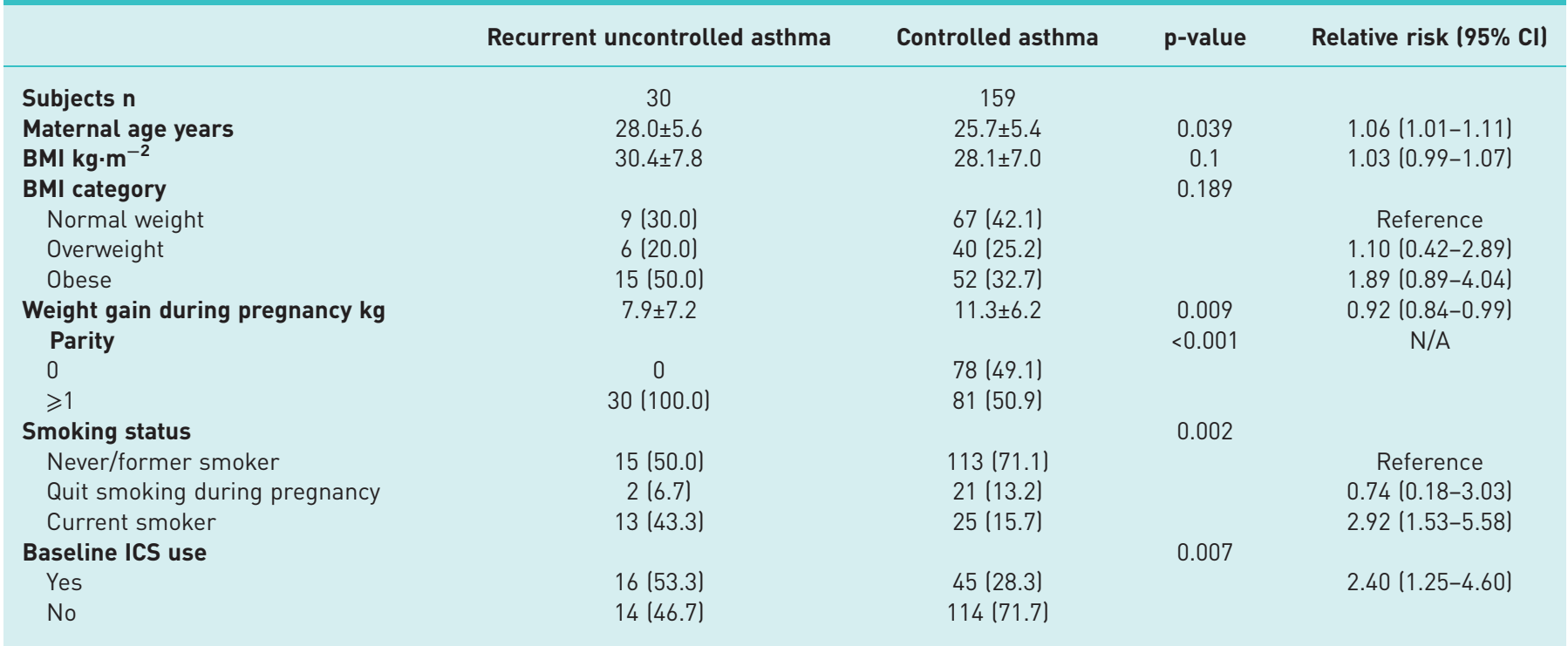

Data are presented as mean \pm SD or $n(\%)$, unless otherwise stated. BMI: body mass index; ICS: inhaled corticosteroid; N/A: not applicable.

worsening asthma in nonpregnant adults [27]. Overweight or obesity may both impact on the airway via similar mechanisms that include systemic inflammation, airway hyperresponsiveness and the mechanical impact of weight itself on thoracic cavity function [28]. The data from this study suggests obesity itself has no impact on the risk of recurrent loss of control, but overweight women are at risk. This may be due to a difference in other risk factors such as smoking between obese and overweight women. Furthermore it

TABLE 5 Relationship between perinatal outcomes and asthma control

\begin{tabular}{cll} 
& Male & \\
\hline $\begin{array}{c}\text { Recurrent } \\
\text { uncontrolled asthma }\end{array}$ & Controlled asthma & p-value
\end{tabular}

\begin{tabular}{cll} 
& \multicolumn{2}{l}{ Female } \\
\hline $\begin{array}{c}\text { Recurrent } \\
\text { uncontrolled asthma }\end{array}$ & Controlled asthma & p-value
\end{tabular}

\section{Subjects $n$}

Gestational age days

Birthweight centile

Birth weight $g$

Delivery method

Vaginal

Elective LSCS

Emergency LSCS

Labour onset

SOL

IOL

None

Birth length $\mathrm{cm}$

Head circumference $\mathrm{cm}$

Preterm birth

No

Yes

SGA

No

Yes

Apgar Score

$1 \mathrm{~min}$

5 min

$\begin{array}{ccc}12 & 85 & \\ 266 \pm 24 & 272 \pm 18 & 0.308 \\ 49.8 \pm 34.1 & 48.0 \pm 31.2 & 0.853 \\ 3280 \pm 761 & 3378 \pm 693 & 0.652 \\ & & 0.578\end{array}$

$9(75.0)$

2 (16.7)

1 (8.3)

$6(50.0)$

4 (33.3)

2 (16.7)

$50.0 \pm 2.0$

$35.5 \pm 1.3$

9 (75.0)

3 (25.0)

$11(91.7)$

$1(8.3)$

$8 \pm 2$

$9 \pm 1$
55 (64.7)

10 (11.8)

20 (23.5)

45 (52.9)

$26(30.6)$

14 (16.5)

$49.8 \pm 3.7$

$34.7 \pm 2.2$

75 (88.2)

75 (88.2)

10 (11.8)

$\begin{array}{ll}8 \pm 1 & 0.395 \\ 9 \pm 1 & 0.805\end{array}$

0.281

0.201

1

0.805
$10(11.8)$

18
$272 \pm 9$
$41.2 \pm 35.5$
$3182 \pm 646$
$9(50.0)$
$6(33.3)$
$3(16.7)$
$6(33.3)$
$6(33.3)$
$6(33.3)$
$48.9 \pm 1.9$
$34.0 \pm 1.8$
$16(88.9)$
$2(11.1)$
$12(66.7)$
$6(33.3)$

$8 \pm 2$
74

$275 \pm 13$

$49.4 \pm 28.5$

0.467

0.302

0.246

0.021

57 (77.0)

7 (9.5)

10 (13.5)

43 (58.1)

$23(31.1)$

$8(10.8)$

$49.3 \pm 2.2$

$34.4 \pm 1.6$
67 (90.5)

7 (9.5)

67 (90.5)

7 (9.5)

$8 \pm 1$

$9 \pm 1$
0.507

0.039

0.348

1

0.018

0.442

0.746

Data are presented as mean \pm SD or $\mathrm{n}(\%)$, unless otherwise stated. LSCS: lower segment caesarean section; SOL: spontaneous onset labour; IOL: induction of labour; SGA: small for gestational age. 
may also be related to dietary intake in this population as we have reported recently that asthmatic women who consumed a diet high in fat, sugar and refined grains were twice as likely to have uncontrolled asthma during pregnancy regardless of BMI [29].

Poor perinatal outcomes associated with maternal asthma appeared to vary with fetal sex. In the current study we observed a greater incidence of SGA female babies in the presence of recurrent uncontrolled asthma. Previous studies have reported a higher incidence of SGA females with the combination of maternal smoking and asthma [30]. Several mechanistic studies have shown that there are sex-specific differences in the fetal response to maternal asthma that may be conferred by a difference in glucocorticoid sensitivity of the male and female placenta [31,32]. These findings suggest males and females respond differently to the presence of maternal asthma and institute different strategies in relation to growth, which may also contribute to difference in perinatal outcomes.

Asthma was not observed to worsen in the presence of a female fetus in the current study, but previous studies have reported a higher incidence of maternal hospitalisations for asthma in women pregnant with a female [33], and reduced maternal lung function and an increased requirement for ICS in women pregnant with a female [7]. However, this remains controversial as large epidemiological studies did not find any difference in maternal exacerbations or daily ICS dose in asthmatic women pregnant with a male or female fetus [34].

While this study was sufficiently powered to assess asthma exacerbations and control during pregnancy, it was underpowered to examine less common perinatal outcomes such as preterm birth. The strengths of this study are reflected in the detailed and prospective collection of data on asthma status and perinatal outcomes. A key challenge related to studies involving asthmatic pregnancies lies in the accurate identification of asthma control status and exacerbations. While validated tools such as the ACQ can be used in the evaluation of asthma control at any point in time [23], no such validated tools are available for identifying exacerbations $[35,36]$. A common approach for defining exacerbations has been to use any event requiring a hospital admission, emergency department presentation, an unscheduled visit to a doctor or a course of oral steroids due to asthma. How well each of these events relates to actual asthma control status remains unclear. For example, women who have greater self-awareness of potentially deteriorating asthma may more readily visit their doctor or the emergency department to seek additional help, while those with poor self-awareness may avoid seeking necessary additional help. This could lead to a situation in which those with greater health-seeking behaviours may appear to experience more exacerbations during pregnancy, but could in turn have better pregnancy outcomes as a result of subsequent improved asthma self-management. In contrast, those with impaired health-seeking behaviours may appear to experience fewer exacerbations, but have worse pregnancy outcomes due to poor asthma self-management. The results of this study in a socially disadvantaged population lend support to these criticisms of the common definition of asthma exacerbations and provide an argument for the preferred use of validated tools such as the ACQ, which does not rely on self-awareness or health-seeking behaviours for assessing asthma-related outcomes during pregnancy. Many previous studies required retrospective collection of exacerbation data from medical records $[10,37,38]$ and we speculate, based our current data, that while exacerbations identified from medical records may be a surrogate marker for persistently poorly controlled asthma during pregnancy, their predictive value in identifying persistently poorly controlled asthma during pregnancy could be biased by the characteristics of the population being studied. Therefore, a major strength of this study is that it provides new evidence that chronically or persistently uncontrolled asthma may be a greater driver for poor perinatal outcomes than an acute exacerbation.

\section{Conclusion}

The current study provides some new and novel insights into the course of asthma during pregnancy, especially the identification that $50 \%$ of all exacerbations and losses of control occur before 20 weeks of gestation. This highlights the need for clinicians to focus on maintaining asthma control during pregnancy through the introduction of asthma education and self-management skills pre-pregnancy to avoid early gestation events that may detrimentally affect placentation and fetal growth. Interestingly, recurrent uncontrolled asthma had the greatest impact on perinatal outcomes, rather than exacerbations, suggesting there is a need for asthma management support during pregnancy to ensure women can maintain asthma control. We speculate that in combination with the identified risk factors for recurrent uncontrolled asthma reported in this paper, which include body weight, cigarette use, ICS use and maternal age, there may also be financial stressors, different purchasing priorities and poor health literacy that could influence ICS use and adherence in this socially disadvantaged population. The key to resolving these issues is to have a more structured approach to asthma education and self-management pre-pregnancy and then a clinically integrated service that encompasses respiratory care with antenatal care. 


\section{Acknowledgements}

The authors would like to acknowledge the valuable contributions made towards the collection and recording of data used in this study by the following individuals: Karen Rogers, Nicolette Hodyl, Natalie Aboustate, Kate Roberts-Thomson, Annette Osei-Kumah, Sarah Riley, Joshua Taverner, Logesh Palanikumar, Thu Nguyen, Zach Stolz, Gordon Goh, and Melissa Liu (University of Adelaide, Adelaide, Australia).

\section{References}

1 Bracken MB, Triche EW, Belanger K, et al. Asthma symptoms, severity, and drug therapy: a prospective study of effects on 2205 pregnancies. Obstet Gynecol 2003; 102: 739-752.

2 Schatz M. Asthma during pregnancy: interrelationships and management. Ann Allergy 1992; 68: 123-133.

3 Schatz M, Dombrowski MP, Wise R, et al. Asthma morbidity during pregnancy can be predicted by severity classification. J Allergy Clin Immunol 2003; 112: 283-288.

4 Murphy VE, Gibson PG, Smith R, et al. Asthma during pregnancy: mechanisms and treatment implications. Eur Respir J 2005; 25: 731-750.

5 Murphy VE, Gibson P, Talbot PI, et al. Severe asthma exacerbations during pregnancy. Obstet Gynecol 2005; 106: 1046-1054.

6 Clifton VL. Sex and the human placenta: mediating differential strategies of fetal growth and survival. Placenta 2010; 31: S33-S39.

7 Murphy VE, Gibson PG, Giles WB, et al. Maternal asthma is associated with reduced female fetal growth. Am J Respir Crit Care Med 2003; 168: 1317-1323.

8 Schatz M, Harden K, Forsythe A, et al. The course of asthma during pregnancy, post partum, and with successive pregnancies: a prospective analysis. J Allergy Clin Immunol 1988; 81: 509-517.

9 Schatz M, Zeiger RS, Hoffman CP, et al. Perinatal outcomes in the pregnancies of asthmatic women: a prospective controlled analysis. Am J Respir Crit Care Med 1995; 151: 1170-1174.

10 Murphy VE, Clifton VL, Gibson PG. Asthma exacerbations during pregnancy: incidence and association with adverse pregnancy outcomes. Thorax 2006; 61: 169-176.

11 Dombrowski MP, Schatz M, Wise R, et al. Asthma during pregnancy. Obstet Gynecol 2004; 103: 5-12.

12 Powell H, Murphy VE, Taylor DR, et al. Management of asthma in pregnancy guided by measurement of fraction of exhaled nitric oxide: a double-blind, randomised controlled trial. Lancet 2011; 378: 983-990.

13 Hodyl NA, Grzeskowiak LE, Stark MJ, et al. The impact of Aboriginal status, cigarette smoking and smoking cessation on perinatal outcomes in South Australia. Med J Aust 2014; 201: 274-278.

14 Hodyl NA, Stark MJ, Scheil W, et al. Perinatal outcomes following maternal asthma and cigarette smoking during pregnancy. Eur Respir J 2014; 43: 704-716.

15 Murphy VE, Clifton VL, Gibson PG. The effect of cigarette smoking on asthma control during exacerbations in pregnant women. Thorax 2010; 65: 739-744.

16 Australian Centre for Asthma Monitoring. Asthma in Australia 2011. AIHW Asthma Series no. 4. Cat. no. ACM 22. Canberra, Australian Institute of Health and Welfare, 2011.

17 Thakur N, Martin M, Castellanos E, et al. Socioeconomic status and asthma control in African American youth in SAGE II. J Asthma 2014; 51: 720-728.

18 Kupczyk M, Haque S, Sterk PJ, et al. Detection of exacerbations in asthma based on electronic diary data: results from the 1-year prospective BIOAIR study. Thorax 2013; 68: 611-618.

19 Curtis LM, Wolf MS, Weiss KB, et al. The impact of health literacy and socioeconomic status on asthma disparities. J Asthma 2012; 49: 178-183.

20 Apter AJ, Wan F, Reisine S, et al. The association of health literacy with adherence and outcomes in moderate-severe asthma. J Allergy Clin Immunol 2013; 132: 321-327.

21 Edwards B, Galletly C, Semmler-Booth T, et al. Antenatal psychosocial risk factors and depression among women living in socioeconomically disadvantaged suburbs in Adelaide, South Australia. Aust N Z J Psychiatry 2008; 42: 45-50.

22 Glover J, Hetzel D, Glover L, et al. A Social Health Atlas of South Australia. Adelaide, Government of South Australia, 2006.

23 Juniper EF, Bousquet J, Abetz L, et al. Identifying 'well-controlled' and 'not well-controlled' asthma using the Asthma Control Questionnaire. Respir Med 2006; 100: 616-621.

24 Gore CJ, Crockett AJ, Pederson DG, et al. Spirometric standards for healthy adult lifetime nonsmokers in Australia. Eur Respir J 1995; 8: 773-782.

25 Blais L, Firoozi F, Kettani FZ, et al. Relationship between changes in inhaled corticosteroid use and markers of uncontrolled asthma during pregnancy. Pharmacotherapy 2012; 32: 202-209.

26 Aanerud M, Carsin AE, Sunyer J, et al. Interaction between asthma and smoking increases the risk of adult airway obstruction. Eur Respir J 2015; 45: 635-643.

27 Schatz M, Zeiger RS, Yang SJ, et al. Prospective study on the relationship of obesity to asthma impairment and risk. J Allergy Clin Immunol Pract 2015; 3: 560-565.

28 Boulet LP. Asthma and obesity. Clin Exp Allergy 2013; 43: 8-21.

29 Grieger JA, Grzeskowiak LE, Wood LG, et al. Asthma control in pregnancy is associated with pre-conception dietary patterns. Public Health Nutr 2016; 19: 332-338.

30 Clifton VL, Hodyl NA, Murphy VE, et al. Effect of maternal asthma, inhaled glucocorticoids and cigarette use during pregnancy on the newborn insulin-like growth factor axis. Growth Horm IGF Res 2010; 20: 39-48.

31 Clifton VL, Davies M, Moore V, et al. Developmental perturbation induced by maternal asthma during pregnancy: the short- and long-term impacts on offspring. J Pregnancy 2012; 2012: 741613.

32 Saif Z, Hodyl NA, Hobbs E, et al. The human placenta expresses multiple glucocorticoid receptor isoforms that are altered by fetal sex, growth restriction and maternal asthma. Placenta 2014; 35: 260-268.

33 Bakhireva LN, Schatz M, Jones KL, et al. Fetal sex and maternal asthma control in pregnancy. J Asthma 2008; 45: 403-407.

34 Firoozi F, Ducharme FM, Lemière C, et al. Effect of fetal gender on maternal asthma exacerbations in pregnant asthmatic women. Respir Med 2009; 103: 144-151.

35 Fuhlbrigge A, Peden D, Apter AJ, et al. Asthma outcomes: exacerbations. J Allergy Clin Immunol 2012; 129: Suppl., S34-S48. 
36 Barranco P, Pérez-Francés C, Quirce S, et al. Consensus document on the diagnosis of severe uncontrolled asthma. J Investig Allergol Clin Immunol 2012; 22: 460-475.

37 Murphy VE, Namazy JA, Powell H, et al. A meta-analysis of adverse perinatal outcomes in women with asthma. BJOG 2011; 118: 1314-1323.

38 Kim S, Kim J, Park SY, et al. Effect of pregnancy in asthma on health care use and perinatal outcomes. $J$ Allergy Clin Immunol 2015; 136: 1215-1223. 